\title{
Recycling old screen-printed electrodes with newly designed plastic antibodies on the wall of carbon nanotubes as sensory element for in situ detection of bacterial toxins in water
}

\author{
Raquel B. Queirós ${ }^{\mathrm{a}, \mathrm{b}, \mathrm{c}}$, A. Guedes ${ }^{\mathrm{d}}$, P.V.S. Marques ${ }^{\mathrm{a}, \mathrm{b}}$, J.P. Noronha ${ }^{\mathrm{e}}$, M.G.F. Sales ${ }^{\mathrm{c}, *}$ \\ a INESC TEC (Coordinated by INESC Porto), Rua do Campo Alegre N. 687, Porto, Portugal \\ b Departamento de Física e Astronomia, Faculdade de Ciências, Universidade do Porto, Porto, Portugal \\ c BioMark/ISEP, Sensor Research, Instituto Superior de Engenharia do Instituto Politécnico do Porto, Portugal \\ d Centro de Geologia da Universidade do Porto and Departamento de Geociências, Ambiente e Ordenamento do Território, Faculdade de Ciências, Universidade do Porto, Portugal \\ e REQUIMTE and Faculdade de Ciências e Tecnologia da Universidade Nova de Lisboa, Portugal
}

\section{A R T I C L E I N F O}

\section{Article history:}

Received 17 September 2012

Received in revised form

27 November 2012

Accepted 30 November 2012

Available online 10 December 2012

\section{Keywords:}

Biosensor

Microcystin-LR

Carbon nanotubes

Potentiometry

Nanomaterials

\begin{abstract}
A B S T R A C T
Using low cost portable devices that enable a single analytical step for screening environmental contaminants is today a demanding issue. This concept is here tried out by recycling screen-printed electrodes that were to be disposed of and by choosing as sensory element a low cost material offering specific response for an environmental contaminant. Microcystins (MCs) were used as target analyte, for being dangerous toxins produced by cyanobacteria released into water bodies. The sensory element was a plastic antibody designed by surface imprinting with carefully selected monomers to ensure a specific response. These were designed on the wall of carbon nanotubes, taking advantage of their exceptional electrical properties. The stereochemical ability of the sensory material to detect MCs was checked by preparing blank materials where the imprinting stage was made without the template molecule.

The novel sensory material for MCs was introduced in a polymeric matrix and evaluated against potentiometric measurements. Nernstian response was observed from $7.24 \times 10^{-10}$ to $1.28 \times 10^{-9} \mathrm{M}$ in buffer solution (10 mM HEPES, $150 \mathrm{mM} \mathrm{NaCl}, \mathrm{pH} 6.6$ ), with average slopes of $-62 \mathrm{mV}_{\text {decade }}{ }^{-1}$ and detection capabilities below $1 \mathrm{nM}$. The blank materials were unable to provide a linear response against $\log$ (concentration), showing only a slight potential change towards more positive potentials with increasing concentrations (while that of the plastic antibodies moved to more negative values), with a maximum rate of $+33 \mathrm{mV}$ decade $^{-1}$. The sensors presented good selectivity towards sulphate, iron and ammonium ions, and also chloroform and tetrachloroethylene (TCE) and fast response $(<20 \mathrm{~s})$. This concept was successfully tested on the analysis of spiked environmental water samples. The sensors were further applied onto recycled chips, comprehending one site for the reference electrode and two sites for different selective membranes, in a biparametric approach for "in situ" analysis.
\end{abstract}

(c) 2012 Elsevier B.V. All rights reserved.

\section{Introduction}

Monitoring environmental contaminants, such as chemical compounds, toxins and pathogens, is crucial to assess and avoid risks for both human and environmental health [1]. Simple, accurate, cost-effective and expeditious methods, enabling to carry out all on-site analytical steps are highly desired in this context. Most of the existing methods combine sophisticated equipment that is unable to meet these requirements, with biosensors at the lead of

\footnotetext{
* Corresponding author at: BioMark Sensor Research/ISEP-IPP, Rua Dr. António Bernardino de Almeida, 431, 4200-072 Porto, Portugal. Tel.: +228 340 500; fax: +228321 159 .

E-mail addresses: goreti.sales@gmail.com, mgf@isep.ipp.pt (M.G.F. Sales).
}

the advantageous alternative methods presented in the literature $[2,3]$.

Biosensors include a biorecognition element coupled to a signal transduction process, many times established in a portable format. A signal is generated when a specific target compound binds to the biorecognition element. Many kinds of biorecognition principles have been adopted so far, including enzymatic, whole cell or affinity-based biosensors [4]. These last ones are very sensitive, selective and versatile, widely recognized as antibodies. These natural materials offer a clear and sensitive response for the target analyte but their degree of selectivity is directly dependent on the way they are produced, which is generally a long, expensive and animal-dependent process [4-8].

Replacing natural by plastic antibodies has been tried out by hundreds of researchers along several decades [9], because they are cheap, fast and easily produced compared to antibodies. This is 
done by tailoring molecular imprinted (MI) materials $[1,10]$ and is still today a great challenge. Great developments have arisen in recent years, by designing the plastic antibodies on the surface of nanomaterials $[11,12]$. This process is called surface imprinting, where a thin polymer film is introduced as an external layer to the nanomaterial support, so that binding sites are at the surface of the polymer and easily accessed by the target molecules [13].

The recent developments among nanomaterials, especially carbon nanostructures have emerged new expectations to the assembly of new affinity-based biorecognition elements. Carbon nanotubes (CNTs) have been found promising materials in this context as they offer high electrical conductivity, chemical stability and mechanical strength, large length-to-diameter ratios, and easy chemical modification with almost any desired chemical or biological specie to their surface [14-17]. They have been included in biosensors as biorecognition elements in different ways. They have been employed as single probes [18], as support of biomaterials $[12,19]$ or individually attached onto a proper transducing surface after synthesis [20]. They may also be used for surface modification or integrated in polymeric matrixes, in both oriented (vertically aligned) and non-oriented (random mixtures) configurations [14]. Several applications of these materials, as part of electrochemical platforms, have also been reported [21-26].

Thus, the main target of the present work is to combine plastic antibody technology and nanomaterials (CNT) within a low-cost recycled platform, enabling to carry out on-site analysis of an environmental contaminant. There are several kinds of environmental contaminants, including drugs and microbial cells and toxins. Much attention has been paid to the tracking of drugs and microbial cells, while microbial toxins are in urgent need of novel and reliable methodologies for in situ determinations.

MCs are a group of highly toxic cyclic heptapeptides produced by several genera of Cyanobacteria, most commonly, Microcystis, Anabaena and Planktothrix [27]. They may display severe hepatotoxic effects, accumulating in the liver and being involved in lipid peroxidation, loss of membrane integrity, DNA fragmentation, apoptosis, cellular disruption, and necrosis [28]. MCs have been responsible for many acute poisonings, including the early reported fatal intoxication of 50 dialysis patients in Brazil in 1996 [29,30].

The most well known toxic MC is MC-LR, where the "Adda" central structure contains Leucine (L) and Arginine in positions 2 and 4 , respectively [31,32]. Its potential chronic toxicity was observed under the form of primary liver cancer, has led the World Health Organization (WHO) to establish a guideline of $1 \mu \mathrm{g} \mathrm{L}^{-1}$ as a maximum concentration of MC-LR in drinking water [33]. MC-LR was also recently classified as a carcinogenic agent by the International Agency for Research on Cancer [34]. The search for fast-screening methods for rapid MCs control requires the development of methods that combine sensitive and selective recognition capabilities of low quantities of analyte.

Potentiometric sensors, a subgroup of chemical sensors, are attractive for practical applications, as they are associated with small size, portability and low energy consumption and cost. Potentiometric sensors operate under conditions of near-zero current flow and measure the difference in potential between the working electrode and a reference electrode, detects the ion activity in the sample and its response is described by the Nernst Equation.

$\mathrm{EMF}=K-\frac{R T}{z F} \ln a_{\mathrm{I}}$

where EMF is the electromotive force (the observed potential at zero current), $K$ is a constant potential contribution that often includes the liquid-junction potential at the reference electrode, $a_{\mathrm{I}}$ is the sample activity for the ion I with charge $z$, and $R, T$, and $F$ are the gas, absolute temperature, and Faraday constant, respectively.
The ion activity describes the analyte concentration, which is often the relevant driving force in chemical or biochemical reactions.

Ion-selective electrodes (ISEs) have become the common method for measuring several ions. In addition, the development of solid contact ISEs along the years has led to major improvements of the limits of detection (LOD), increased range of applications, and simplification of the sensor construction, operation, and maintenance [35-38].

Herein, the design of a novel plastic antibody for MC-LR on the surface of CNTs and the integration of this material over a recycled screen-printed electrode coupled to potentiometric transduction are presented. The ability of the sensory material to selectively recognize MC-LR and its cross-reactivity against other MCs is shown, along with the overall analytical features of the device.

\section{Materials and methods}

\subsection{Apparatus}

All potential measurements were made by a Crison decimilivoltammeter, pH meter, GLP $21( \pm 0.1 \mathrm{mV}$ sensitivity) at room temperature and under constant stirring. The assembly of the potentiometric cell was: conductive solid support | MC-LR-selective membrane | buffered sample solution (10 mM 4-(2-hydroxyethyl)1-piperazineethanesulfonic acid (HEPES), $150 \mathrm{mM} \mathrm{NaCl}, \mathrm{pH}$ 6.6) ॥ electrolyte solution, $\mathrm{KCl}|\mathrm{AgCl}| \mathrm{Ag}$ (s). The reference electrode was $\mathrm{Ag}|\mathrm{AgCl}| \mathrm{KCl}$ saturated reference electrode from Crison Instrument, S.A. The selective electrode was prepared in conventional configuration for batch mode evaluations. These devices had no internal reference solution and epoxy-graphite as solid contact [39]. The membrane was applied on the top of the carbon-paste electrodes. When necessary, the $\mathrm{pH}$ was measured by a Consort combined glass electrode connected to the Crison decimilivoltammeter, pH meter, GLP 21.

Polymeric film depositions over the metal contacts of the screen-printed electrode have been carried out by using an Autolab PGSTATION 128N computerized electrochemical instrument, controlled by dedicated NOVA 1.6 software (Ecochemie, Utrecht, The Netherlands). A $1 \mathrm{~mm} \times 5 \mathrm{~mm}$ silver electrode has been used as working electrode, while a glassy carbon rod and a pseudo silver electrode have been used as counter and reference electrode, respectively.

Scanning electron microscopic (SEM) images and energydispersive X-ray spectroscopy (EDS) analysis were collected by a FEI Quanta 400FEG ESEM/EDAX Genesis X4M microscope. Raman experiments were performed using a JOBIN-YVON LABRAM spectrometer, and a He-Ne laser was used giving a monochromatic red light of $632.8 \mathrm{~nm}$ at a power of $20 \mathrm{~mW}$. Raman spectra were measured with a hole of $1000 \mu \mathrm{m}$ and a slit of $100 \mu \mathrm{m}$. A 50x air objective lens of an Olympus optical microscope was used to focus the laser beam on the sample and also to collect the scattered radiation. A highly sensitive CCD camera was used to collect the Raman spectra. Extended scans from $100 \mathrm{~cm}^{-1}$ to $3500 \mathrm{~cm}^{-1}$ were performed on each sample.

\subsection{Reagents and solutions}

All chemicals were of analytical grade and double-deionized RNAse free water $0.03 \mu \mathrm{S}$ at $25^{\circ} \mathrm{C}$ from ATS (Portugal) was used in the preparation of all solutions. MC-LR, multi-wall CNTs (CNTs, $>7.5 \%$, basis O.D. $\times \mathrm{L} \quad 7-15 \mathrm{~nm} \times 0.5-10 \mu \mathrm{m}$ ), sodium 4-vinylbenzenesulfonate (VBSate), vinylbenzoate (VBate), tris(2-methoxyethoxy)vinylsilane (TMVS), benzoyl peroxide (BOP), vinylbenzene (VB), 3,4-ethylenedioxythiophene (EDOT), HEPES and divinylbenzene (DVB) were purchased from Sigma, 


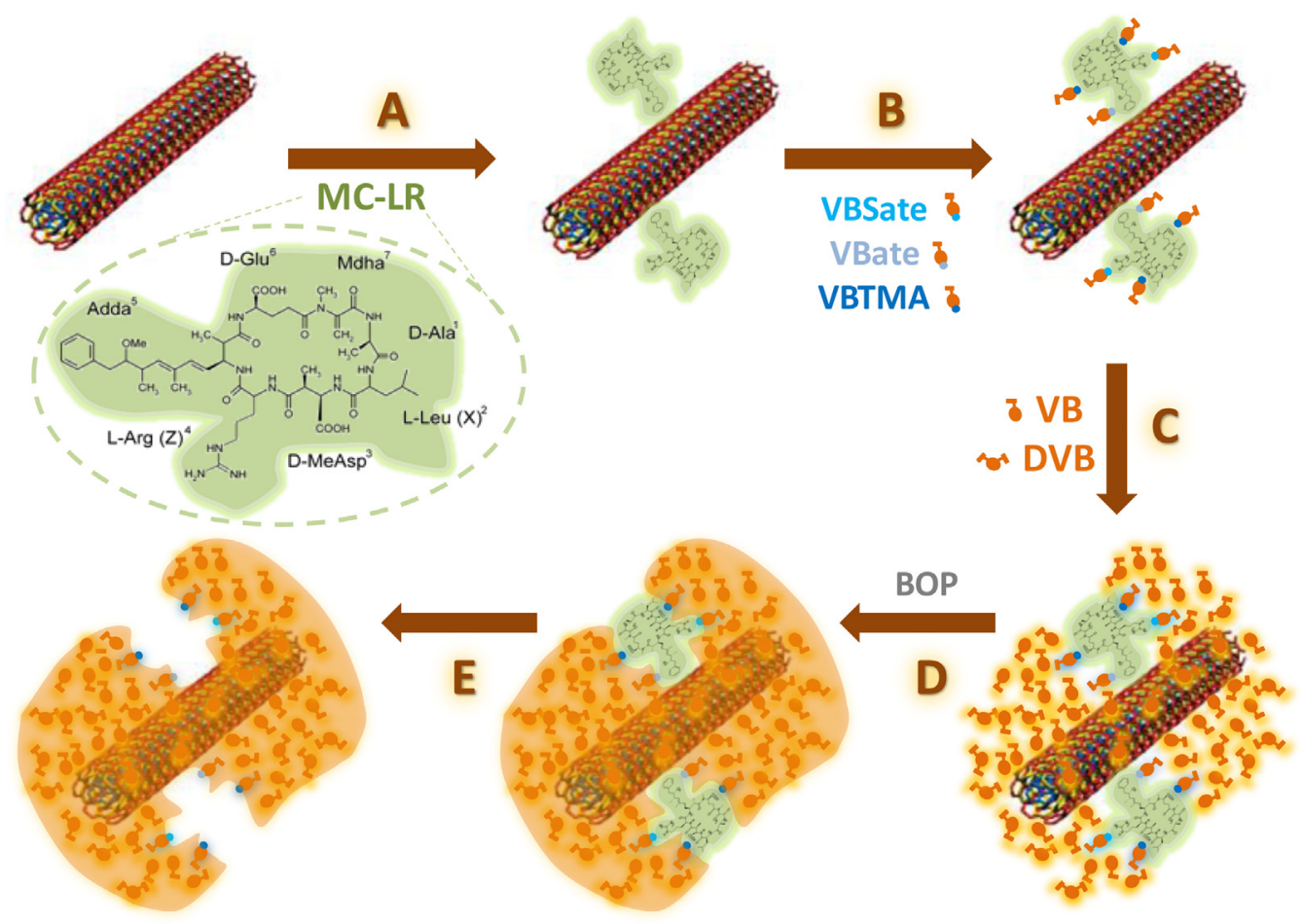

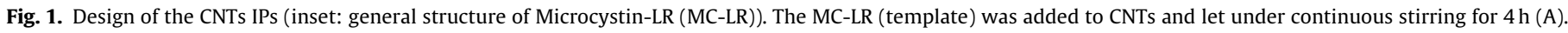

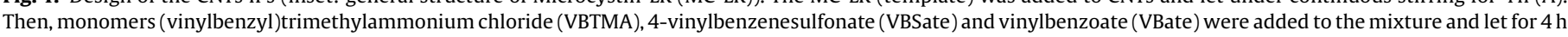

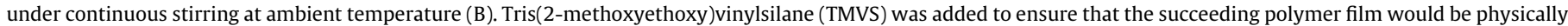

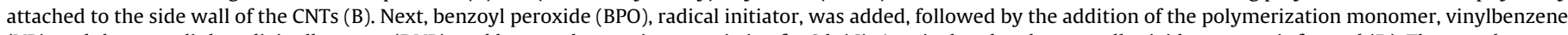

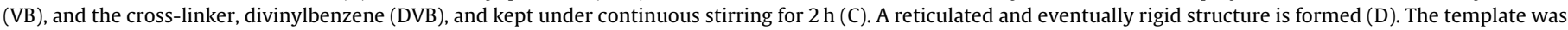
extracted by washing thoroughly with fresh buffer solution (E).

Europe. Potassium tetrakis(4-chlorophenyl)borate (TpCIPB) and onitrophenyl octyl ether (oNPOE) were obtained from Acros, Spain. PVC of high molecular weight, (vinylbenzyl)trimethylammonium chloride (VBTMA), potassium sulfate were purchased from Fluka, Europe, and tetrahydrofuran (THF) was obtained from Riedel-deHäen, Germany. Sodium chloride was purchased from Panreac, Spain. Chloroform was purchased by Carlo Erba, Europe. Iron III chloride 6-hydrate was purchased by Scharlau, Spain. Ammonium acetate was purchased by Analar, Portugal. TCE was obtained from Merck, Germany. The buffer used was $10 \mathrm{mM}$ HEPES, $150 \mathrm{mM} \mathrm{NaCl}$ with a $\mathrm{pH}$ of 6.6 .

\subsection{Synthesis of host-tailored polymers}

The chemical structure of MC-LR has both polar and apolar regions. It contains three possible charged positions ( 2 carboxylic acids and 1 amine) and a side chain that may establish hydrophobic interactions. This hydrophobic side of the structure was used to adsorb MC-LR to the outer wall of the CNTs, while the eminent charged sites were used to attract charged monomers that, being involved in the subsequent polymerization process, would create charged binding sites for MC-LR that were spatially distributed in complementary positions. Almost all chemical changes were carried out under mild conditions to ensure that the 3D arrangement and electrostatic environment of MC-LR were preserved.

To prepare the molecular imprinted carbon nanotubes (MICNTs) biomaterial, MC-LR (template, $3.5 \mathrm{mmol}$ methanol) was added to $2 \mathrm{mg}$ of CNTs and let under continuous stirring for $4 \mathrm{~h}$ (Fig. 1A). Then, VBTMA (monomer, $7 \mathrm{mmol}$ ), VBSate (monomer, $3.5 \mathrm{mmol}$ ) and VBate (monomer, $3.5 \mathrm{mmol}$ ), all carrying charged organic functional groups, were added to this mixture and let for $4 \mathrm{~h}$ under continuous stirring at ambient temperature (Fig. 1B). Ionic interactions between these monomers and the charged groups of the adsorbed MC-LR were established here; the position of the vinyl groups in these monomers was conditioned by the charges of MC-LR and remained free to participate in the subsequent polymerization step, responsible for imprinting the target molecule. After, the mixture was centrifuged and the supernatant removed. The solid material was let stand for $1 \mathrm{~h}$ at $40^{\circ} \mathrm{C}$ to evaporate the remaining supernatant. To ensure that the succeeding polymer film would be physically attached to the side wall of the CNTs (which would prevent the plastic antibody layer from being lixiviated to the liquid-phase), $100 \mu$ L of TMVS was added and let stand (Fig. 1B). Sol-gel chemistry ensured that the CNTs were covalently bound to the silane group and the vinyl group remained free and capable of participating in the polymerization step. CNTs contain hydroxyl, carbonyl and carboxylic groups at its surface enabling the formation of covalent bounds between these groups and CNTs. In particularly, hydroxyl groups act as binding sites in the silanization process [40-42]. To ensure that the polymeric layer is covalently attached to CNTs is not necessary the full coverage of the entire CNTs surface but only a few anchor points.

Next, BPO (radical initiator, $6 \mathrm{mg}$ ) was added, followed by the addition of VB (monomer, $50 \mu \mathrm{L}$ ) and DVB (cross-linker, $50 \mu \mathrm{L}$ ), kept under continuous stirring for $2 \mathrm{~h}$ (Fig. 1C). These benzene derivative monomers have only phenyl radicals linked to the vinyl group. The presence of these phenyl groups was expected to increase the electron density of the polymer film, contributing to the easy polarization of this material. DVB acted as cross-linker because it carried two vinyl groups, leading to the formation of a reticulated and eventually rigid structure (Fig. 1D). This polymerization was carried out at room temperature for only $2 \mathrm{~h}$, in order to avoid any alterations of the MC-LR conformation. Then the mixture was centrifuged and the supernatant was removed. The mixture was then thoroughly washed with 3 cycles of centrifugation and re-suspension in fresh buffer solution, to washout 
Table 1

Selective membrane composition and the corresponding analytical features in a buffer of $10 \mathrm{mM} \mathrm{HEPES} \mathrm{and} 150 \mathrm{mM} \mathrm{NaCl}$, $\mathrm{pH} 6.6$ ( $n=3$ ).

\begin{tabular}{|c|c|c|c|c|c|c|c|c|c|}
\hline \multirow[t]{2}{*}{ Sensor } & \multirow[t]{2}{*}{ Ionophore } & \multicolumn{4}{|c|}{ Composition } & \multirow[t]{2}{*}{ Linear range $(\mathrm{M})$} & \multirow{2}{*}{$\begin{array}{l}\text { Slope } \\
\left(\mathrm{mV} \text { decade }^{-1}\right)\end{array}$} & \multirow[t]{2}{*}{ LOD (M) } & \multirow[t]{2}{*}{$r^{2}$} \\
\hline & & $\begin{array}{l}\text { Sensor } \\
(\mathrm{mg})\end{array}$ & $\begin{array}{l}\text { oNPOE } \\
(\mathrm{mg})\end{array}$ & $\begin{array}{l}\text { TpClPB } \\
(\mathrm{mg})\end{array}$ & $\begin{array}{l}\text { PVC } \\
(\mathrm{mg})\end{array}$ & & & & \\
\hline I & MI & 0.5 & 30 & - & 15 & $1.32 \times 10^{-9}$ to $7.75 \times 10^{-10}$ & $-62 \pm 4$ & $6.92 \times 10^{-10} \pm 2.8 \times 10^{-11}$ & 0.998 \\
\hline II & MI + additive & 0.5 & 30 & 0.25 & 15 & $1.21 \times 10^{-9}$ to $7.41 \times 10^{-10}$ & $-53 \pm 6$ & $6.61 \times 10^{-10} \pm 1.0 \times 10^{-11}$ & 0.996 \\
\hline III & $\mathrm{NI}$ & 0.5 & 30 & - & 15 & $1.28 \times 10^{-9}$ to $7.24 \times 10^{-10}$ & $29 \pm 5$ & $5.25 \times 10^{-10} \pm 1.0 \times 10^{-11}$ & 0.981 \\
\hline IV & Additive & - & 30 & 0.25 & 15 & - & - & - & - \\
\hline V & Blank & - & 30 & - & 15 & $1.19 \times 10^{-9}$ to $6.84 \times 10^{-10}$ & $31 \pm 3$ & $6.35 \times 10^{-10} \pm 1.0 \times 10^{-10}$ & 0.984 \\
\hline
\end{tabular}

LOD - limit of detection.

exceeding reactants and the template (Fig. 1E). Finally, the obtained material was recovered by filtration and let to dry overnight under nitrogen atmosphere inside a desiccator. Non-imprinted carbon nanotubes (NI-CNTs) biomaterial was also prepared in parallel, by the same process but excluding the template from the overall process. In this case, the charged monomers do not interact with the template molecule (not present here) and the cavities are not form. The polymer form by VB, BPO and DVB is formed only around CNTs.

\subsection{Preparation of sensory membranes}

Five sensing membranes were prepared with the composition presented in Table 1. The core MC-LR selective membranes (Sensor I) were prepared by mixing MI-CNTs (ionophore, $0.50 \mathrm{mg}$ ) with PVC (polymer, $15.0 \mathrm{mg}$ ) and oNPOE (plasticizer, $30.0 \mathrm{mg}$ ). An additional membrane having also TpCIPB (anionic additive, $0.25 \mathrm{mg}$ ) was also prepared (Sensor II). Three additional membranes were made in parallel as control: one had NI sensory material as ionophore (Sensor III) and the other two had no ionophore (Sensor IV and V) with one of these including the additive (Sensor IV).

Each of the above mixtures was stirred until the PVC was well moistened, and dispersed in $0.5 \mathrm{~mL}$ THF. These membranes were coated on carbon-electrodes and let to dry during $48 \mathrm{~h}$. After drying, the electrodes were kept in buffer solution. The regeneration of the electrodes was made by washing the membranes in ultrapure water for 30 min between measurements.

\subsection{Potentiometric measurements}

Several selective membranes were prepared by including MI and NI materials in plasticized PVC and casting the resulting solutions on solid-state carbon supports. Their overall composition was indicated in Table 1. Decreasing concentration levels of MC-LR were obtained by transferring increasing aliquots of buffer solution, $\mathrm{pH}$ 6.6, to $10.0 \mu \mathrm{L}$ of $5 \times 10^{-6} \mathrm{M}$ of MC-LR. The range of concentrations studied was $4.58 \times 10^{-10}$ to $2.00 \times 10^{-9} \mathrm{M}$. The potential readings of the stirred MC-LR solutions were measured in buffer at room temperature and recorded after stabilization to $\pm 1 \mathrm{mV}$, according to IUPAC recommendations [43].

\subsection{Selectivity studies}

Other species that are common in waters or expected as contaminant may interfere with the response of the potentiometric device. Selectivity studies followed the Matched Potential Method (MPM) [44]. The change in EMF caused by the principal ion was measured for a MC-LR rise in concentration from $1.0 \times 10^{-6} \mathrm{M}$ to $5.0 \times 10^{-6} \mathrm{M}$ using a MI-CNT sensor. Similarly, the EMF of a $1.0 \times 10^{-6}$ M MC-LR solution was measured against these species up to their maximum admitted levels and a solution of interfering species added until the same potential change was observed. Solutions, prepared in buffer solution, of $0.5 \mathrm{mg} \mathrm{L}^{-1} \mathrm{NH}_{4}{ }^{+}, 250 \mathrm{mg} \mathrm{L}^{-1} \mathrm{Cl}^{-}, 250 \mathrm{mg} \mathrm{L}^{-1} \mathrm{SO}_{4}{ }^{2-}$, $200 \mu \mathrm{gL}^{-1} \mathrm{Fe}^{3+}, 200 \mathrm{mg} \mathrm{L}^{-1} \mathrm{Na}^{+}, 100 \mu \mathrm{gL}^{-1} \mathrm{CHCl}_{3}$ and $10 \mu \mathrm{gL}^{-1}$ of TCE were tested for this purpose, being these the main components/contaminants in waters by the Portuguese Law [45].

\subsection{Analysis of environmental waters}

Environmental water samples were collected in an artesian well and stored in amber glass bottles previously rinsed with doubledeionized RNase free water. The $\mathrm{pH}$ and ionic strength of the water samples was adjusted by addition of buffer (50:50), and they were stored at $4{ }^{\circ} \mathrm{C}$ until analysis. The samples were finally spiked with MC-LR. These analyses were carried out in duplicate.

\subsection{Recycling and designing the chips}

Used screen-printed electrodes (SPEs) (Fig. 5) were obtained from an old/non-reusable commercial screen-printed electrode carrying three silver contacts $(200 \mathrm{~mm} \times 5 \mathrm{~mm})$ on a ceramic substrate $(200 \mathrm{~mm} \times 100 \mathrm{~mm})$ and recycled in order to enable their application in the in situ analysis of environmental waters, using only $\mu$ volumes of samples. The ceramic support was cut to remove the indicating, reference and counter electrode support material. The insulating polymer on top of these silver contacts was removed to expose the conductive silver and allow their use as conductive support. One of the sides of the ceramic was used as reference (RE) and working (WE) electrodes while the other side was used as electrical contact in the switch box where these screen-printed electrodes are routinely used. A $10^{-2} \mathrm{M} \mathrm{FeCl}_{3} \cdot 6 \mathrm{H}_{2} \mathrm{O}$ solution was deposited on the silver track acting as RE to allow the formation of a silver chloride on top of it. Each WE was first modified with a poly(EDOT) layer, by potentiostatic electropolymerization of EDOT (monomer, $0.01 \mathrm{M}$ ), at a potential of $+0.9 \mathrm{~V}$ during $240 \mathrm{~s}$, in a supporting electrolyte of $0.1 \mathrm{M} \mathrm{KClO}_{3}$. The deposited polymer was then reduced at $-0.5 \mathrm{~V}$ for $60 \mathrm{~s}$ in the same electrolyte solution and subsequently stabilized by cyclic voltammetry. For this purpose, 5 subsequent potential scans, from -0.5 to $+0.5 \mathrm{~V}$, at a potential scan rate of $0.05 \mathrm{~V} \mathrm{~s}^{-1}$ were recorded in the same supporting electrolyte solution [46]. The ceramic support was then let dry and the selective membranes casted on top of each WE track. These membranes were physically separated, an essential condition to obtain independent potentials. The potentiometric measurements were made in buffer.

\section{Results and discussion}

\subsection{SEM and EDS analysis}

SEM analysis was conducted over CNT, MI-CNT and NI-CNT to confirm the formation of a polymeric layer around the nanotubes of carbon. The obtained images are shown in Fig. 2, along with the corresponding EDS spectra. Plain CNTs were composed by carbon only, as indicated in the EDS spectra. The EDS analysis shows that polymers are formed around CNTs. In the EDS analysis of CNTs only carbon (C) is observed, while in MI-CNTs and NI-CNTs 


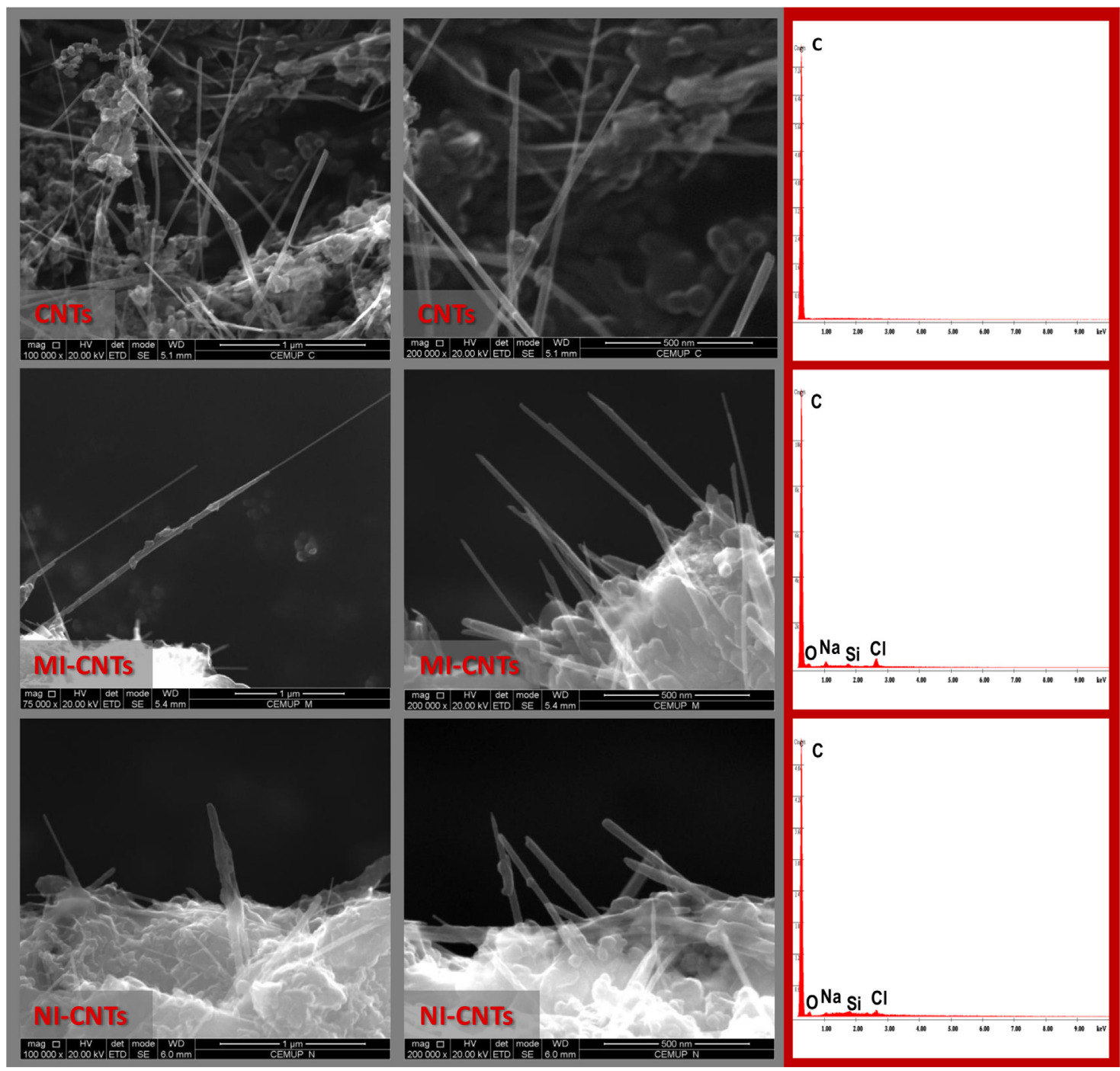

Fig. 2. SEM (left) and EDS (right) analysis of (A) CNTs, (B) MI-CNTs, (C) NI-CNTs (direct sample analysis over dry materials).

other components due to the polymer formation around CNTs were observed. The tubes showed 8-20 nm thickness, being this wide range a consequence of using multi-wall nanotubes.

Modified CNTs (MI or NI) displayed thicker tubes, ranging within 18 and $40 \mathrm{~nm}$ (Fig. 2). When compared to pure CNTs, the resolution of the SEM images of these materials under higher amplification $(40,0000 \times)$, was reduced accounting for a decrease in the conductivity of this material. This observation supported the existence of organic carbon-based structures over the tubes of carbon. Technical differences between MI and NI materials are however not clear under electron microscopy evaluations, with further studies being needed.

\subsection{Raman analysis}

The obtained Raman spectra for CNTs, MI-CNTs and NI-CNTs are shown in Fig. 3. The spectra of CNTs showed three main peaks: (i) the $\mathrm{D}$ band at $1338 \mathrm{~cm}^{-1}$, originated from the $\mathrm{sp}^{3}$-hybridized carbons on the nanotubes; (ii) the $G$ band at $1575 \mathrm{~cm}^{-1}$, attributed to the a splitting of the $E_{2 g}$ stretching mode of graphite-like structures, which is relevant to vibration of the $\mathrm{sp}^{2}$-bonded carbon atoms in a two-dimensional hexagonal lattice; and (iii) the 2D band at $2657 \mathrm{~cm}^{-1}$, assigned to the overtone of the D band. The two most important of these bands were the $G$ and the $D$ band, which represented the $\mathrm{sp}^{2}$ and $\mathrm{sp}^{3}$ structures [47]. The $\mathrm{G}$ band corresponded to the $E_{2 g}$ modes which represented the movement in opposite directions of two neighbouring carbon atoms in a graphite sheet. The D band was attributed to the disorder present in the CNTs.

The intensity ratio $\mathrm{D} / \mathrm{G}$ band is often used to monitor the functionalization of CNTs [48]. In first-order Raman spectra, the positions of the bands $\mathrm{D}, \mathrm{G}$ and $\mathrm{D}^{\prime}\left(1100-1700 \mathrm{~cm}^{-1}\right)$ presented very similar behaviour, however the intensity ratio $D / G$ ratio showed differed behaviours for each material which may be correlated to differences in their structural ordering and CNTs diameter $[49,50]$. As can been seen in the Raman spectra (Fig. 3), the intensity ratio of CNTs is higher (0.7) than the modified ones (MI-CNTs and NI-CNTs), which presented values of 0.31 and 0.32 , respectively. The increase of tube diameter of the imprinted CNTs is the most probable responsible for the decrease on the ratio D/G observed on MI-CNTs and NI-CNT and may be correlated to a reduction on defect density. This is also the reason for the $D / G$ ratio of unmodified CNTs is smaller. This corroborated well the presence of a polymeric layer on the overall CNTs structures. In addition, a small feature called G2 or D' near $1610 \mathrm{~cm}^{-1}$ was observed in the modified CNTs, characteristic of large diameter distribution of the CNTs [51].

Full-width half-maximum (FWHM) in the Raman spectrum has been considered as a criterion in evaluating the structural disorder of CNTs, which arises from bond angle and bond length distortions [52]. The FWHM of the D-band (FWHM (D)) of modified CNTs (MICNTs and NI-CNTs) is narrowed than the CNTs. This is consistent 


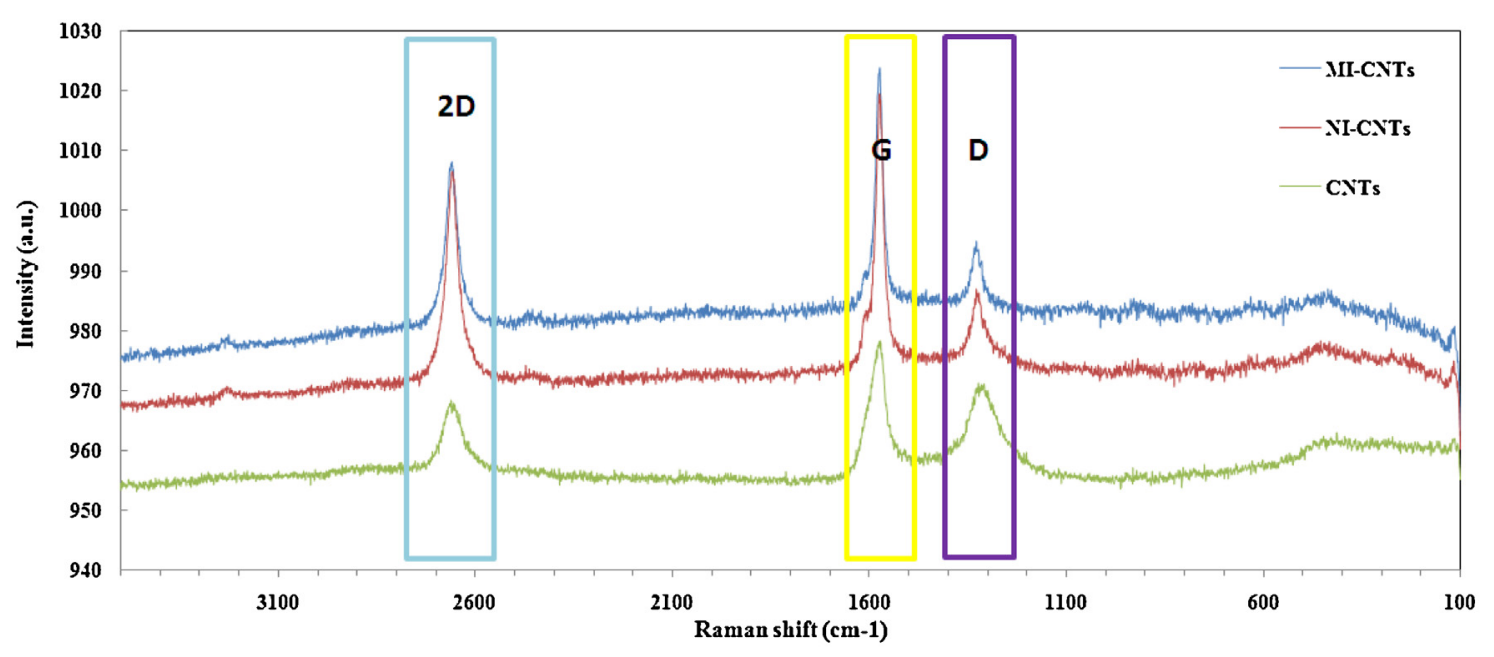

Fig. 3. Raman spectra of the CNTs, MI-CNTs and NI-CNTs.

with the introduction of a polymeric matrix which causes disorder bonds and amorphous carbon since FWHM (D) has the same tendency as the intensity ratio $\mathrm{D} / \mathrm{G}[50,53]$.

Furthermore, $\mathrm{D}$ bands of modified CNTs appear with a significant shift to the CNTs. While the D band shifted 20 and $21 \mathrm{~cm}^{-1}$ in MICNTs and NI-CNTs materials, respectively, the G band shifted 4 and $3 \mathrm{~cm}^{-1}$. In general, these shifts have been attributed to the structural changes introduced on the material organization by growing a polymeric layer over the CNTs.

Typical approaches to use Raman spectroscopy for assessment of purity have relied upon the intensity ratio of $D / G$. Recently, a report from DiLeo et al., was published where the ratio using the 2D band peak is shown to represent a more accurate alternative for measuring MWCNT quality and purity [54]. The results show that 2D band of the modified CNTs (MI-CNTs and NI-CNTs) was more intense than simple CNTs. This mode is known to be sensitive to increasing defect density. Second-order mode of 2D increases in intensity with increase in defects [55], which can be consistent with the polymerization around the CNTs materials.

Overall, the obtained results confirmed the existence of slight structural differences between MI-CNTs and NI-CNTs materials and major differences between these and the CNTs.

\subsection{Evaluation of standard curves}

MI-CNTs and NI-CNTs presented, surprisingly, opposite behaviour (Fig. 4A). Membranes with MI-CNTs (I and II) displayed negative slopes, while membranes with NI-CNTs (III) showed positive ones (Table 1 ). This opposite potential change against MC concentration was attributed solely to the differences in the CNT modified materials, mostly because membranes (I) and (III) had only these materials acting as ionophores included in a plasticized PVC membrane. In addition, a blank membrane of plasticized PVC (V) was unable to provide a potentiometric response.

When a lipophilic anionic additive was included in MI-CNT membranes (II), the slope kept (surprisingly) its negative value. This additive was included in a small amount to increase the permselectivity of the membrane without governing the response of the electrode. This was not a good choice because the presence of the additive deteriorated the analytical features of the final device, exhibiting sub-Nernstian behaviour. This behaviour was consistent with the presence of charged sites within the imprinted cavities, not requiring additional charges to acquire a Nernstian response. A membrane with only additive in plasticized PVC (V) was also prepared as control and was unable to produce a potentiometric response, at least within the concentration range studied.

In general, the biomimetic sensors presented good reproducibility $(n=3)$, fast response $(t \approx 20 \mathrm{~s})$, good sensitivity, linear dynamic range and low limits of detection, in all cases below the guideline value ( $1 \mathrm{nM}$ ) established by WHO [33].

\subsection{Selectivity against organic and inorganic compounds}

There are several approaches to assess potentiometric selectivity, expressed in terms of potentiometric selectivity coefficient $\left(K_{\mathrm{IJ}}^{\mathrm{pot}}\right)$, where I is the main ion and $\mathrm{J}$ the foreign one. Separated solutions, mixed solutions or matched potential methods are typically employed for this purpose. Technically, the later one is the only method enabling the evaluation of neutral species, accounting for a more realistic approach and being applicable to sensors that do not show Nernstian responses. The values of $K_{\mathrm{IJ}}^{\text {pot }}$ were calculated from equation (Eq. (1)), where $a_{\mathrm{J}}$ was the interfering concentration required to give the same potential variation as the concentration of $a_{\mathrm{I}}$ (both added over a common background of $\mathrm{I}$ ).

$K_{\mathrm{IJ}}^{\mathrm{pot}}=\frac{\Delta a_{\mathrm{I}}}{a_{\mathrm{J}}(\mathrm{IJ})}$

Almost none of the tested species was able to provide the potential change of the primary ion solution, indicating that the electrodes displayed a very high selectivity for MC-LR. Only chloride and sodium managed to meet the desired potential change, but only when these were in much higher concentration than MC-LR. The average $\log \left(K_{\mathrm{MC}-\mathrm{LR}, \mathrm{Cl}}^{\mathrm{pot}}\right)$ and $\log \left(K_{\mathrm{MC}-\mathrm{LR}, \mathrm{Na}}^{\mathrm{pot}}\right)$ was -3.3 and -3.2 , respectively, indicating that the response to MC-LR prevailed. Thus, the electrodes showed very good selectivity properties for MC-LR, indicating that these were suitable for the analysis of environmental waters.

\subsection{Cross-response}

There are several parent MCs in environmental waters that show close structural similarities. The cross-response of the sensor against these is therefore an important analytical parameter [56]. This should provide some information about the way the analytical signal should be expressed: either the sensor turns out specific for MC-LR and all sample analytical data is attributed to its content in MC-LR; or it gives a similar response to most of the MCs and the 

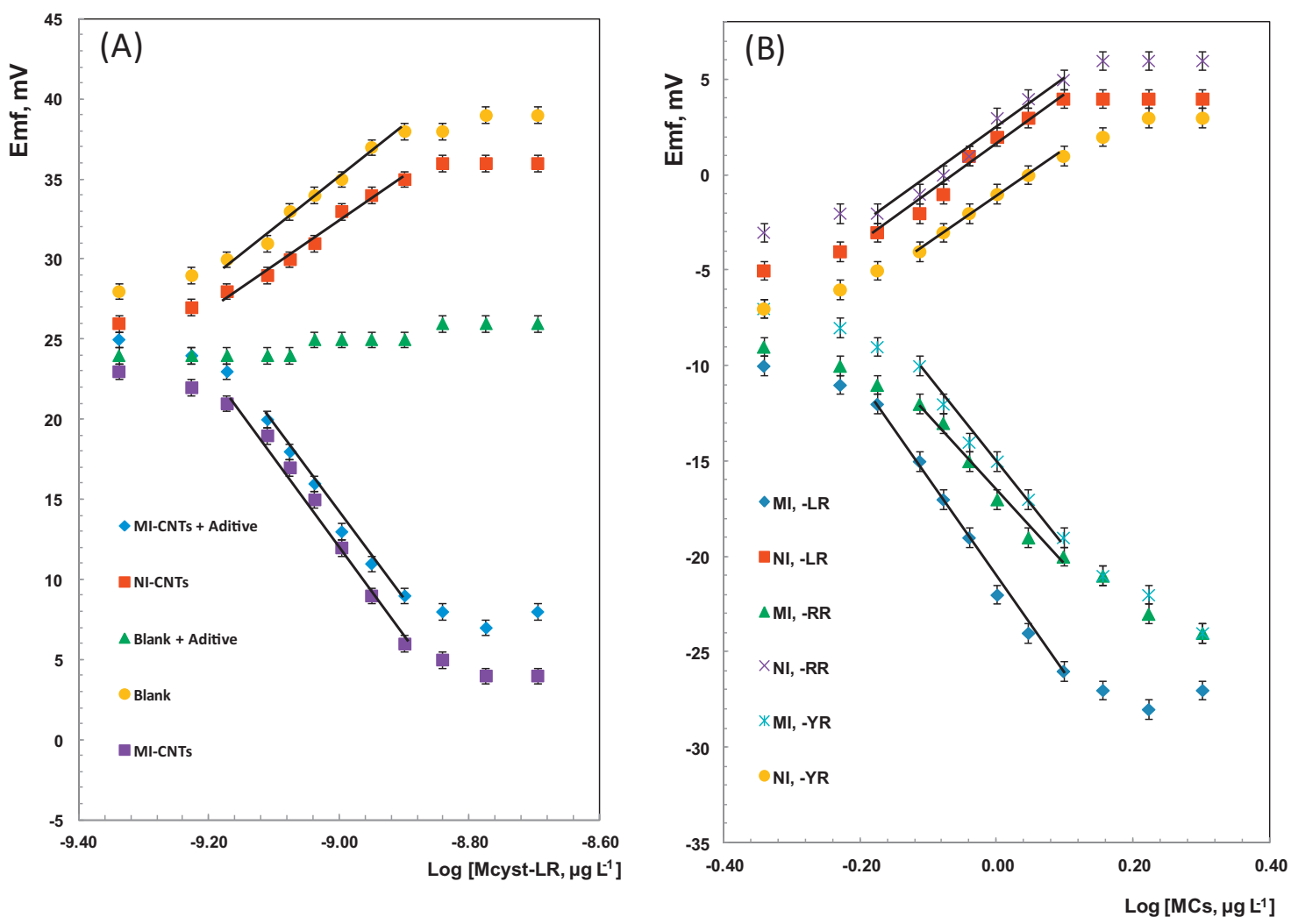

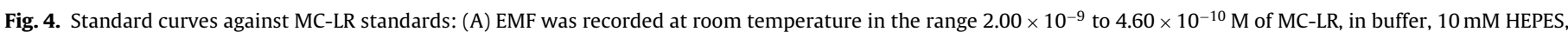
$150 \mathrm{mM} \mathrm{NaCl}, \mathrm{pH}$ 6.6; and their cross-reactivity against other MCs (B), MC-LR, MC-YR and MC-RR also in buffer, $10 \mathrm{mM}$ HEPES, $150 \mathrm{mM}$ NaCl, pH 6.6.

resulting data must be interpreted as a combined result of all MCs expressed in terms of MC-LR standards.

The response of MI and NI sensors for MC-LR, MC-YR and MC-RR are presented in Fig. 4B. The results showed that MI sensors presented similar behaviour for all MCs as well as NI sensors presented similar behaviour for all MCs. Thus, close similarities between these MCs full the sensory material, making the analytical response to MCs in environmental waters a measure of the total amount of MCs present.

\subsection{Analysis in a biparametric disposable chip for in situ measurements}

The chips presented a linear behaviour (squared correlation coefficients $>0.991$ ) in the same range of concentration used previously $\left(2.00 \times 10^{-9}\right.$ to $4.58 \times 10^{-10} \mathrm{M}$ of MC-LR) and with the same positive/negative slopes (Fig. 6). A significant difference was observed however, as the signal amplified, up to slopes of -216 and $205 \mathrm{mV} \mathrm{decade}^{-1}(n=2)$ for MI-CNTs and NI-CNTs, respectively.
(B)

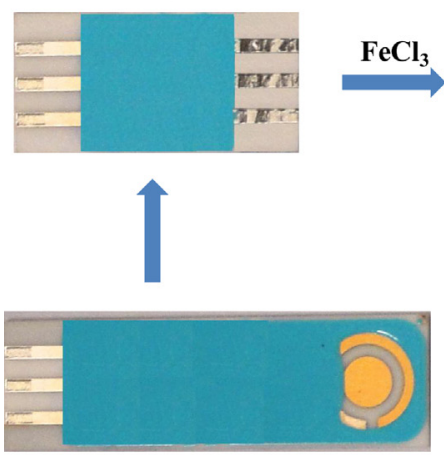

(C)

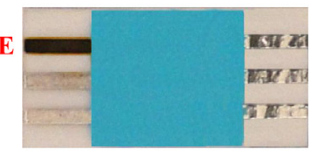

(A)

Used Screen-printed electrode
(D)

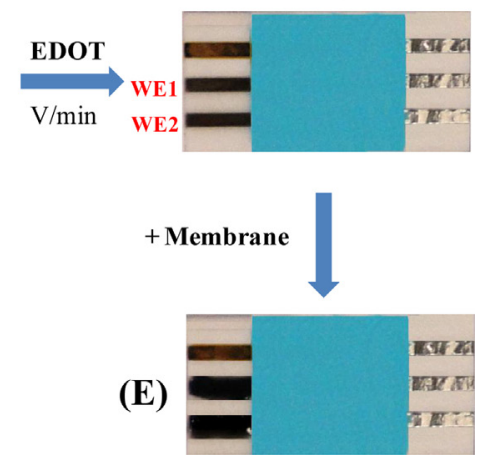

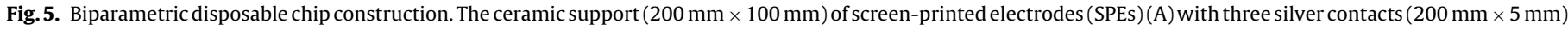

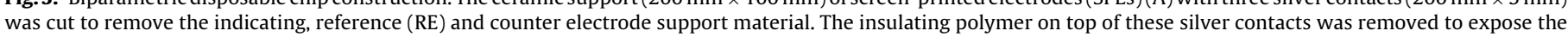

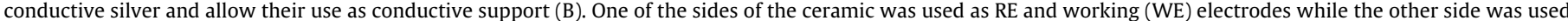

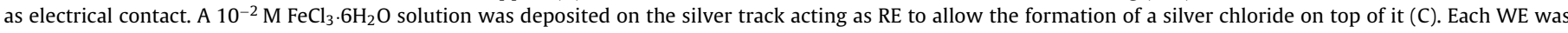

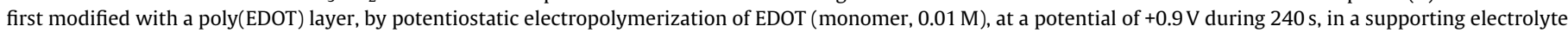

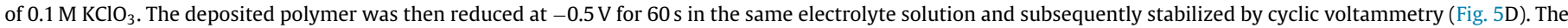

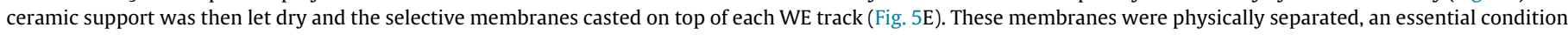
to obtain independent potentials. The potentiometric measurements were made in buffer. 


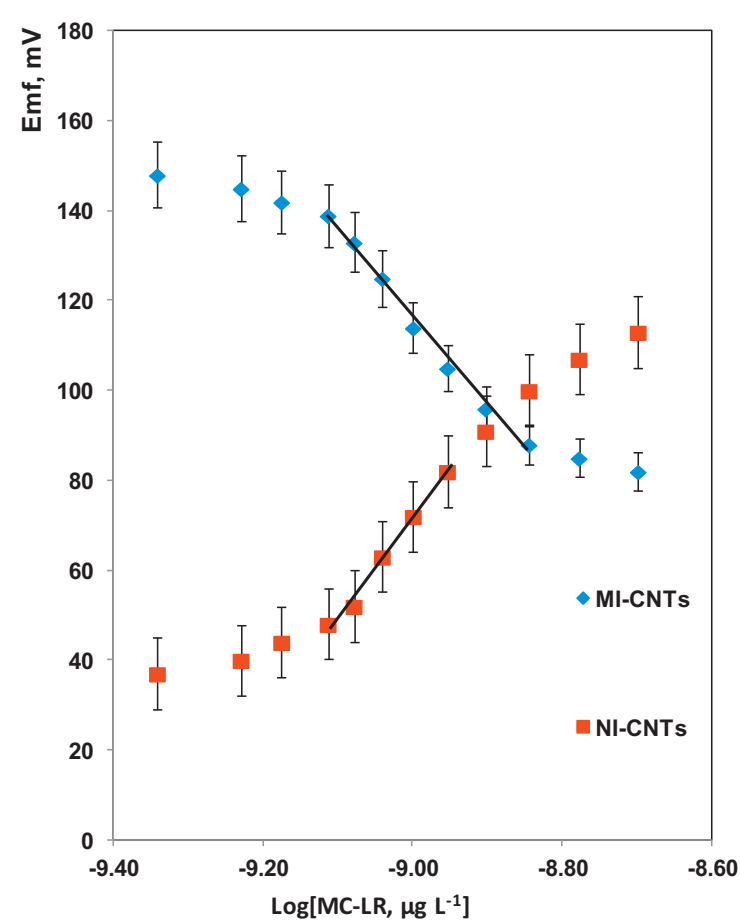

Fig. 6. Standard curves against MC-LR standards for SPEs, EMF was recorded at room temperature in the range $2.00 \times 10^{-9}$ to $4.60 \times 10^{-10} \mathrm{M}$ of MC-LR, in buffer, $10 \mathrm{mM}$ HEPES, $150 \mathrm{mM} \mathrm{NaCl}$, pH 6.6.

The use of these chips allowed the simultaneous measurements of 2 different membranes but the concept is easily extended to as many contacts are placed in the chip. The contacts are connected to a derivation box that read the signals individually without any interference between the signals. The use of these kinds of systems allows the reduction of the sample needed, up to $0.5 \mathrm{~mL}$, and enable the analysis in situ.

\subsection{Application to environmental waters}

In order to investigate the practical application of the present method, several waters were spiked and analyzed, and the corresponding relative errors calculated. These assays were conducted in environmental waters ( $\mathrm{pH}$ 6.5) with conventional sensors (MICNTs and NI-CNTs) calibrated within $2 \times 10^{-9}$ to $4.6 \times 10^{-10} \mathrm{M}$ of MC-LR. The average recoveries of two spiked solutions was $102.4 \pm 1.7 \%$, with an average relative error of $-2.4 \%$, thus confirming the accuracy of the analytical data ( $n=2$ for two sensors). The results were precise, with relative standard deviation $<6 \%$.

\section{Conclusions}

A particularly successful surface imprinting approach was established to produce biomimetic materials for MC-LR used under potentiometric transduction, capable of differentiating imprinted from non-imprinted materials. In general, the sensors offered design simplicity, short measuring time, good precision, high accuracy, low limit of detection (below the guideline value established by WHO) and good selectivity to inorganic and organic compounds. The analytical data provided by the sensors should be correlated to all MCs in the sample, with a biparametric disposable SPE enabling in situ measurements. Further developments may be achieved by increasing the number of electrical contacts in the chip for more efficient multi-analyte performance.

\section{Acknowledgement}

Raquel B. Queirós thanks Fundação para a Ciência e Tecnologia, for the PhD grant SFRH/BD/49072/2008.

\section{References}

[1] B. Van-Dorst, J. Mehta, K. Bekaert, E. Rouah-Martin, W.D. Coen, P. Dubruel, R. Blusta, J. Robbens, Recent advances in recognition elements of food and environmental biosensors: a review, Biosensors and Bioelectronics 26 (2010) 1178-1194.

[2] S. Rodriguez-Mozaz, M.J. Lopez de Alda, D. Barceló, Biosensors as useful tools for environmental analysis and monitoring, Analytical and Bioanalytical Chemistry 386 (2006) 1025-1041.

[3] A.J. Baeumner, Biosensors for environmental pollutants and food contaminants, Analytical and Bioanalytical Chemistry 377 (2003) 434-445.

[4] E. Luzi, M. Minunni, S. Tombelli, M. Mascini, New trends in affinity sensing-aptamers for ligand binding, Trends in Analytical Chemistry 22 (11) (2003) 810 .

[5] A.E.G. Cass, Biosensors: A Practical Approach, IRL Press, Oxford, 1990.

[6] K. Mosbach, O. Ramström, The emerging technique of molecular imprinting and its future impact on biotechnology, Nature Biotechnology 14 (1996) 163.

[7] A.P.F. Turner, I. Karube, G.S. Wilson, Biosensors: Fundamentals and Applications, Oxford University Press, Oxford, 1987.

[8] G. Wulff, Molecular imprinting in cross-linked materials with the aid of molecular templates-a way towards artificial antibodies, Angewandte Chemie International Edition 34 (1995) 1812.

[9] K. Haupt, K. Mosbach, Molecularly im-printed polymers and their use in biomimetic sensors, Chemical Reviews 100 (2000) 2495-2504.

[10] Y. Hoshino, T. Kodama, Y. Okahata, K.J. Shea, Peptide imprinted polymer nanoparticles: a plastic antibody, Journal of the American Chemical Society 130 (2008) 15242-15243.

[11] Y. Hoshino, H. Koide, T. Urakami, H. Kanazawa, T. Kodama, N. Oku, K.J. Shea, Recognition, Neutralization, and clearance of target peptides in the bloodstream of living mice by molecularly imprinted polymer nanoparticles: a plastic antibody, Journal of the American Chemical Society 132 (2010) 6644-6645.

[12] F.T.C. Moreira, R.A.F. Dutra, J.P. Noronha, A.L. Cunha, M.G.F. Sales, Artificial antibodies for troponin $\mathrm{T}$ by its imprinting on the surface of multiwalled carbon nanotubes: its use as sensory surfaces, Biosensors and Bioelectronics 28 (2011) 243-250.

[13] C.-L. Choong, J.S. Bendall, W.I. Milne, Carbon nanotube array: a new MIP platform, Biosensors and Bioelectronics 25 (2009) 652-656.

[14] K. Balasubramanian, M. Burghard, Biosensors based on carbon nanotubes, Analytical and Bioanalytical Chemistry 385 (2006) 452-468.

[15] A. Merkoçi, M. Pumera, X. Llopis, B. Pérez, M. del Valle, S. Alegret, New materials for electrochemical sensing VI-carbon nanotubes, Trends in Analytical Chemistry 24 (2005) 826-838.

[16] W. Yang, P. Thordarson, J.J. Gooding, S.P. Ringer, F. Braet, Carbon nanotubes for biological and biomedical applications, Nanotechnology 18 (2007) 412001 (12 pp.).

[17] J.J. Gooding, R. Wibowo, J. Liu, W. Yang, D. Losic, S. Orbons, F.J. Mearns, J.G. Shapter, D.B. Hibbert, Protein electrochemistry using aligned carbon nanotubes electrode arrays, Journal of the American Chemical Society 125 (2003) 9006-9007.

[18] F.N. Ishikawa, B. Stauffer, D.A. Caron, C. Zhou, Rapid and label-free cell detection by metal-cluster-decorated carbon nanotube biosensors, Biosensors and Bioelectronics 24 (2009) 2967-2972.

[19] Y. Mao, Y. Bao, S. Gan, F. Li, L. Niu, Electrochemical sensor for dopamine based on a novel graphene-molecular imprinted polymers composite recognition element, Biosensors and Bioelectronics 28 (2011) 291-297.

[20] J. Li, J.E. Koehne, A.M. Cassell, H. Chen, H. Tee, Q. Ye, W. Fan, J. Han, M. Meyyappan, Inlaid multi-walled carbon nanotube nanoelectrode arrays for electroanalysis, Electroanalysis 17 (2005) 15-27.

[21] S. Carrara, V.V. Shumyantseva, A.I. Archakov, B. Samorì, Screen-printed electrodes based on carbon nanotubes and cytochrome P450scc for highly sensitive cholesterol biosensors, Biosensors and Bioelectronics 24 (2008) 148-150.

[22] X. Lu, J. Zhou, W. Lu, Q. Liu, J. Li, Carbon nanofiber-based composites for the construction of mediator-free biosensors, Biosensors and Bioelectronics 23 (2008) 1236-1243.

[23] M. Pan, G. Fang, Z. Duan, L. Kong, S. Wang, Electrochemical sensor using methimazole imprinted polymer sensitized with MWCNTs and Salen-Co(III) as recognition element, Biosensors and Bioelectronics 31 (2012) 11-16.

[24] M.A. Poggi, L.A. Bottomley, P.T. Lillehei, Scanning probe microscopy, Analytica Chemistry 74 (2002) 2851-2862.

[25] L. Tang, Y. Zhu, X. Yang, J. Sun, C. Li, Self-assembled CNTs/CdS/dehydrogenase hybrid-based amperometric biosensor triggered by photovoltaic effect, Biosensors and Bioelectronics 24 (2008) 319-323.

[26] M. Zhang, A. Smith, W. Gorski, Carbon nanotube-chitosan system for electrochemical sensing based on dehydrogenase enzymes, Analytical Chemistry 76 (2004) 5045-5050.

[27] W.W. Carmichael, S.M.F.O. Azevedo, J.S. An, R.J.R. Molica, E.M. Jochimsen, S. Lau, K.L. Rinehart, G.R. Shaw, G.K. Eagelsham, Cyanobacteria: chemical and biological evidence for cyanotoxins, Environmental Health Perspectives 109 (2001) 663-668. 
[28] T. Chen, Q. Wang, J. Cui, W. Yang, Q. Shi, Z. Hua, J. Ji, P. Shen, Induction of apoptosis in mouse liver by microcystin-LR, Molecular and Cellular Proteomics 4 (2005) 958-974.

[29] S. Pouria, A. Andrade, J. Barbosa, R.L. Cavalcanti, V.T.S. Barreto, C.J. Ward, W. Preiser, G.K. Poon, G.H. Neild, G.A. Codd, Fatal microcystin intoxication in haemodialysis unit in Caruaru, Brazil, Lancet 352 (1998) 21-26.

[30] T. Kuiper-Goodman, S. Gupta, H. Combley, B.H. Thomas, Microcystins in drinking water: risk assessment and derivation of a possible guidance value for drinking water, in: D.A. Steffensen, B.C. Nicholson (Eds.), Toxic Cyanobacteria-A Global Perspective, Australian Centre for Water Quality Research, Salisbury, 1994, pp. 17-23.

[31] M.M. Gehringer, P. Milne, F. Lucietto, T.G. Downing, Comparison of the structure of key variants of microcystin to vasopressin, Environmental Toxicology and Pharmacology 19 (2005) 297-303.

[32] J. Meriluoto, B. Kincaid, M.R. Smyth, M. Wasberg, Electrochemical detection of microcystins, cyanobacterial peptide hepatotoxins, following highperformance liquid chromatography, Journal of Chromatography A 810 (1998) 226-230.

[33] World Health Organization (WHO), Guidelines for Drinking-Water Quality, vol. 2, 2nd ed., WHO, Geneva, 1998.

[34] Y. Grosse, R. Baan, K. Straif, B. Secretan, F. El Ghissassi, V. Cogliano, Carcinogenicity of nitrate, nitrite, and cyanobacterial peptide toxins, Lancet Oncology 7 (2006) 628-629.

[35] S. Singh, A. Srivastava, H.-M. Ohb, C.-Y. Ahnb, G.-G. Choib, R.K. Asthana, Recent trends in development of biosensors for detection of microcystin, Toxicon 60 (5) (2012) 878-894.

[36] E. Bakker, E. Pretsch, Potentiometric sensors for trace-level analysis, Trends in Analytical Chemistry 27 (2007) 612-618.

[37] E. Bakker, E. Pretsch, Potentiometric sensors for trace-level analysis, Trends in Analytical Chemistry 24 (2005) 199-207.

[38] S.A. Piletsky, A.P.F. Turner, Electrochemical sensors based on molecularly imprinted polymers, Electroanalysis 14 (2002) 317-323.

[39] A.H. Kamel, M.G.F. Sales, S.A.A. Almeida, F.T.C. Moreira, Novel potentiometric sensors of molecular imprinted polymers for specific binding of chlormequat, Analytical Sciences 25 (2009) 365-371.

[40] B. Scheibe, E. Borowiak-Palen, R.J. Kalenczuk, Oxidation and reduction of multiwalled carbon nanotubes-preparation and characterization, Materials Characterization 61 (2010) 185-191.

[41] J. Kathi, K.Y. Rhee, Surface modification of multi-walled carbon nanotubes using 3-aminopropyltriethoxysilane, Journal of Material Science 43 (2008) 33-37.

[42] J.H. Luong, S. Hrapovic, D. Wang, F. Bensebaa, B. Simard, Solubilization of multiwall carbon nanotubes by 3-aminopropyltriethoxysilane towards the fabrication of electrochemical biosensors with promoted electron transfer, Electroanalysis 16 (2004) 132-139.

[43] R.P. Buck, V.V. Cosofret, Recommended procedures for calibration of ionselective electrodes, Pure and Applied Chemistry 65 (1993) 1849-1858.

[44] Y. Umezawa, K. Umezawa, H. Sat, Selectivity coefficients for ion-selective electrodes: recommended methods for reporting $K_{(\mathrm{pot}, \mathrm{a}, \mathrm{b})}$ values, Applied Chemistry 67 (1995) 507-518.

[45] Decreto-Lei 307/2007 (Portuguese Law)-Republic Journal 1st Serie 164 (2007) 5747.

[46] A. Bello, M. Giannetto, G. Mori, R. Seeber, F. Terzi, C. Zanardi, Optimization of the DPV potential waveform for determination of ascorbic acid on PEDOT-modified electrodes, Sensors \& Actuators B-Chemistry 121 (2007) 430-435.

[47] H.S. Hsu, P.Y. Chung, J.H. Zhang, S.J. Sun, H. Chou, H.C. Su, C.H. Lee, J. Chen, J.C.A Huang, Observation of bias-dependent low field positive magneto-resistance in Co-doped amorphous carbon films, Applied Physics Letters 97 (2010), 032503.

[48] C. Gao, Y.Z. Jin, H. Kong, R.L.D. Whitby, S.F.A. Acquah, G.Y. Chen, H. Qian, A. Hartschuh, S.R.P. Silva, S. Henley, P. Fearon, Polyurea-functionalized multiwalled carbon nanotubes: synthesis, morphology, and Raman spectroscopy, Journal of Physical Chemistry B 109 (2005) 11925-11932.

[49] E.F. Antunes, A.O. Lobo, E.J. Corat, V.J. Trava-Airoldi, A.A. Martin, C. Veríssimo, Comparative study of first- and second-order Raman spectra of MWCNT at visible and infrared laser excitation, Carbon 44 (2006) 2202-2211.

[50] E.F. Antunes, A.O. Lobo, E.J. Corat, V.J. Trava-Airoldi, A.A. Martin, C. Veríssimo, Influence of diameter in the Raman spectra of aligned multi-walled carbon nanotubes, Carbon 45 (2007) 913-921.
[51] M. Zdrojek, W. Gebicki, C. Jastrzebski, T. Melin, A. Huczko, Studies of multiwall carbon nanotubes using Raman spectroscopy and atomic force microscopy, Solid State Phenomena 99 (2004) 265-268.

[52] C. Casiraghi, A.C. Ferrari, J. Robertson, Raman spectroscopy of hydrogenated amorphous carbons, Physical Review B 72 (2005) 085401.

[53] H. Liu, Y. Zhang, R. Li, X. Sun, F. Wang, Z. Ding, P. Mérel, S. Desilets, Aligned synthesis of multi-walled carbon nanotubes with high purity by aerosol assisted chemical vapor deposition: effect of water vapour, Applied Surface Science 256 (2010) 4692-4696.

[54] R.A. DiLeo, B.J. Landi, R.P. Raffaelle, Purity assessment of multiwalled carbon nanotubes by Raman spectroscopy, Journal of Applied Physics 101 (6) (2007) 064301-1-064301-5

[55] N. Chakrapani, S. Curran, W. Bingqing, P.M. Ajayan, A. Carrillo, R.S. Kane, Spectral fingerprinting of structural defects in plasma treated carbon nanotubes, Journal of Materials Research 18 (10) (2003) 2515-2521.

[56] T. Kawaguchi, D.R. Shankaran, S.J. Kim, K.V. Gobi, K. Matsumoto, K. Toko, N. Miura, Fabrication of a novel immunosensor using functionalized selfassembled monolayer for trace level detection of TNT by surface plasmon resonance, Talanta 72 (2007) 554-560.

\section{Biographies}

Raquel B. Queirós graduated in 2007 in Chemical Engineering (Technologies for Environmental Protection) and in 2008 a master degree in Chemical Engineering from Instituto Superior de Engenharia do Instituto Politécnico do Porto. Currently, she is concluding her Ph.D. in Physics at University of Porto in collaboration with associate laboratory INESC TEC and BioMark/ISEP on the development of biosensors for detection of aquatic bacterial contamination. Her interests included the synthesis and characterization of new biomimetics materials for electrochemical and optical analytical applications. She is a student member of International Society for Optical Engineers (SPIE) and President of the University of Porto SPIE Student Chapter.

Alexandra Guedes received a Ph.D. in Geology at University of Porto in 2001. She is a researcher at Faculty of Sciences, University of Porto with interests in the characterization of materials by Raman microspectroscopy.

Paulo Marques was born in Coruche, Portugal, in 1968. He received a degree in Physics (1991), a M.Sc. in Optoelectronics and Lasers (1995) and a Ph.D. in Physics (2000), all from the University of Porto, Portugal. Currently he is an Assistant Professor at the Physics and Astronomy Department at Porto University. Since July 2000 he develops research activity in the Optoelectronics and Electronics Systems Unit (UOSE) of INESC PORTO, where it has been responsible for several Nationa and European research contracts. His current research interests include hybrid sol-gel based waveguides, optical photosensitivity, Bragg gratings, integrated optical sensors, optofluidics and laser direct writing techniques for integrated optics and microfabrication in general. He has published 4 world patents (patent family of 32 ) and published more than 100 scientific papers in international magazines and conferences and two book chapters. Since October 2009 is the coordinator of the Optoelectronics and Electronics Systems Unit of INESC Porto.

João Paulo Noronha graduated with a degree in Pharmaceutical Sciences from the University of Porto, Porto, Portugal, in 1987, and the Ph.D. degree in Organic Chemistry from the Nova University of Lisbon, in 2001, research performed at the Chemistry Department of the Faculty of Sciences and Technology of the Nova University of Lisbon, on chromatographic hyphenated techniques. His main research interests are in the chromatographic and mass spectrometric analysis of environmental, pharmaceutical, proteomic areas. He is an Assistant Professor the Chemistry Department of the Faculty of Sciences and Technology of the Nova University of Lisbon.

Goreti Sales received a degree in Pharmaceutical Sciences at the Faculty of Pharmacy of Porto University in 1994 and a Ph.D. in Analytical Chemistry at the Porto University in 2000. She is a full professor in the Polytechnique Institute, School of Engineering and mainly devoted to the research of biomimetic nanomaterials. 